\title{
Review Article \\ Significance of Kampo, Traditional Japanese Medicine, in Supportive Care of Cancer Patients
}

\section{Jun-ichi Yamakawa, ${ }^{1}$ Yoshiharu Motoo, ${ }^{2}$ Junji Moriya, ${ }^{1}$ Masao Ogawa, ${ }^{3}$ Hiroaki Uenishi, Sumiyo Akazawa, ${ }^{1}$ Toshiyuki Sasagawa, ${ }^{4}$ Matomo Nishio, ${ }^{5}$ and Junji Kobayashi ${ }^{1}$}

\author{
${ }^{1}$ Department of General Medicine, Kanazawa Medical University, 1-1 Daigaku, Uchinada, Ishikawa 920-0293, Japan \\ ${ }^{2}$ Department of Medical Oncology, Kanazawa Medical University, 1-1 Daigaku, Uchinada, Ishikawa 920-0293, Japan \\ ${ }^{3}$ Department of Anesthesiology, Kanazawa Medical University, 1-1 Daigaku, Uchinada, Ishikawa 920-0293, Japan \\ ${ }^{4}$ Department of Obstetrics and Gynecology, Kanazawa Medical University, 1-1 Daigaku, Uchinada, Ishikawa 920-0293, Japan \\ ${ }^{5}$ Department of Pharmacology, Kanazawa Medical University, 1-1 Daigaku, Uchinada, Ishikawa 920-0293, Japan
}

Correspondence should be addressed to Jun-ichi Yamakawa; yamakawa@kanazawa-med.ac.jp

Received 19 March 2013; Accepted 28 May 2013

Academic Editor: Sookyung Lee

Copyright (C) 2013 Jun-ichi Yamakawa et al. This is an open access article distributed under the Creative Commons Attribution License, which permits unrestricted use, distribution, and reproduction in any medium, provided the original work is properly cited.

\begin{abstract}
The current standard treatment for cancer is a multidisciplinary therapy whereby various types of treatment are properly combined. Chemotherapy with multiple anticancer drugs is now common, and traditional, complementary, and alternative therapies are adopted as supportive measures. Medical care in Japan is distinguished by the ability for patients to access both Western and Kampo medical cares at the same time. There is a high degree of trust in the safety of Kampo therapies because they are practiced by medical doctors who are educated with fundamental diagnosis of Western medicine. Highly reliable clinical studies are being published, demonstrating that palliative or supportive care for cancer patients using Kampo preparations alleviates adverse effects of chemotherapy or radiotherapy. This paper reports the circumstances around cancer care in Japan where traditional therapeutic Kampo formulas are used for patients undergoing cancer treatment with cutting-edge chemotherapy, specifically to alleviate adverse effects of anticancer drugs.
\end{abstract}

\section{Background}

1.1. Historical Background of Cancer Treatment in Japan. Surgery, radiotherapy, and chemotherapy are the main medical treatments for cancer. Chief among those is surgery. In recent years, advances have been made in a range of treatments that target specific characteristics and stages of cancer. By its nature, cancer develops after gene mutations in the body's cells, and the difficulty in treating cancer lies in the fact that cells metastasize. Surgery and radiation are local therapies, which leave the problem of how to treat the invisible remaining cancer cells. What is then required is not a localized treatment but a systemic treatment such as chemotherapy.

But until progress was made in the development of anticancer drugs, there was no effective treatment against cancer once it had spread throughout the body. A combination of surgery with chemotherapy is generally used. And sometimes radiotherapy is also used. Nowadays, the standard treatment is "multidisciplinary treatment [1-5]," a comprehensive form of treatment that efficiently combines a variety of treatments.

1.2. Anticancer Drugs. Chemotherapy now occupies an important position in the treatment of cancer. Anticancer drugs have greatly changed cancer treatment. Excellent therapeutic effects have recently been achieved by combining radiation with anticancer drugs, even for solid cancers. The Achilles heel of anticancer drugs has been the strength of the adverse reactions [6-18]; however, these have been alleviated with the development of administration methods and supportive care to control nausea, vomiting, and so forth; therefore, patients do not suffer as much as before. Yet, the history of chemotherapy is still short. Surgery has been available for about 100 years and radiotherapy for about 50 , 
but anticancer drugs have only been used to treat cancer for the last 35 years.

Anticancer drugs have completely different effects depending on the type of cancer. While chemotherapy may be effective for some cancers, it is virtually ineffective for others. The effects of anticancer drugs also differ according to the way they are used. Potent effects are demonstrated when using drugs in combination, even if each anticancer drug does not promise sufficient effect when used alone. Nowadays, two to four types of anticancer drugs are used in combination to enhance their effectiveness, even at a modest amount. Such multidrug therapy is now being widely used and offers the hope of synergistic or additive effects.

1.3. Historical Background of Kampo Medicine. Traditional, complementary, and alternative therapies [19-25] are widely used and researched in the USA. Underlying this is the high cost of health care in that country and the common use of cheap folk remedies as well as traditional therapies and supplements against illness. The same situation exists in Europe and is becoming more widespread in Asia, where governments are promoting integrative medicine. There is a universal health insurance system which enables everybody in Japan to receive advanced health care at low cost. Therefore, alternative medicine did not attract attention. Japan's universal health insurance system $[26,27]$ is held in high regard across the country, and it means patients receive standard care for cancer at any medical service provider under this insurance system. However, if you prefer complementary or alternative therapies, you must pay a private provider out of your own pocket.

Yet, another characteristic of medical care in Japan is that patients can access Western and Kampo medical cares at the same time. Kampo medicine [28-30] is a unique medical system that originated from ancient China, was gradually imported to Japan since approximately 1500 years ago, and has been improved and refined by many excellent physicians especially since the 17 th century (Edo periods in Japanese era). Now, most Kampo preparations (Japanese traditional herbal medicines) are available as extract formulations of high quality, which are greatly different from herbal medicines used in China, Taiwan, and Korea, where most preparations are herbal decoctions.

Four ethical Kampo extract formulations were approved in 1967 in Japan. Since then, the number of ethical Kampo extract formulations covered by health insurance has grown to 148. Much Japanese herbal extract preparation is used in Japan. Kampo extract preparation is mostly used in Japan. These Kampo extracts are the combination of herbal medicines from the viewpoint of Kampo theory. Standard examinations are done, and quality control of index ingredient is displayed. Kampo formulation for prescription is used for cancer medical treatment. Japan's universal health insurance system does allow simultaneous access to traditional Kampo preparations and Western medicines. However, doctors in Japan cannot be licensed without passing a board examination of Western medicine, which means patients in this country receive health care with a high degree of safety. This is another factor that distinguishes the health care system in Japan from other countries. In Japan, physicians who have studied Western medicine and Kampo medicine practice these approaches in their medical treatment of cancer with the aim of fusing Eastern and Western medicines into a unitary medical system, unlike the dual medical systems in China or Korea.

\subsection{Supportive Care for Cancer Patients Using Kampo Prepa-} rations. Some people involved in the treatment of cancer reject Kampo therapy. The biggest reason they give is the scarcity of evidence. Kampo medicine is fundamentally a tailor-made type of treatment, and Kampo prescription is changed according to the patients' condition and symptoms. Therefore, the benefits of Kampo preparations cannot be fully evaluated using the criteria of the randomized clinical trials as in Western medicine. Objective data and proof of action mechanisms are required. Most of the studies on the actions of Kampo preparations have been animal trials and smallscale clinical trials. Little research has been done that offers highly reliable evidence, although progress has been made in this area recently [31-37]. The use of Kampo preparations for palliative and supportive care of cancer patients in combination with anticancer drugs or radiotherapy may offer alleviation of adverse effects and survival benefits, and the number of such research papers being published in international journals is increasing.

\section{Kampo for Chemotherapy-Induced Peripheral Neuropathy}

2.1. Cancer Chemotherapy-Induced Peripheral Neuropathy. A drawback of most anticancer drugs currently in use is that they are not cancer cell-specific: their actions affect all multiplying cells. They interfere with cancer cell division by interfering with DNA replication and the functioning of the proteins necessary for cell division, but they also damage normal cells. Myeloid cells, immune cells, gastrointestinal mucosal cells, and hair root cells are particularly susceptible to damage and are prone to adverse effects such as bone marrow suppression, immunodeficiency, digestive symptoms, and alopecia. Since nerve and muscle cells do not undergo cell division, they are thought to be robust against such damage. However, some anticancer drugs are known to cause peripheral neuropathy. While it is only certain anticancer drugs that has this side effect, we know that patients who take the following drugs develop peripheral neuropathy: taxanebased drugs [38-43] such as paclitaxel and docetaxel; vinca alkaloids such as vincristine sulfate; and platinum-based drugs [41, 44-55] including cisplatin and oxaliplatin. The causes involve injury to axonal microtubules and direct injury to nerve cells. Microtubules are necessary for the transfer of chromosomes when cells divide. If the formation of microtubules is disturbed, cell division is inhibited. In addition, microtubules are also found within axons, which transmit nerve cell signals, and are involved in axonal development and material transportation. Vinca alkaloids and taxanes, in particular, act on the microtubules within cancer cells but cause neuropathy because they simultaneously damage the 
microtubules in normal nerve cells. Platinum-based drugs directly damage nerve cells and are thought to lead to nerve cell axon disorder.

\subsection{Medical Treatment of Peripheral Neuropathy. Peripheral} neuropathy symptoms include limb extremity numbness, as well as sensory motor ataxia, deep tendon reflex decline, and decreased muscle strength. There is great variation among sufferers of such complaints because sensation of these symptoms is extremely subjective. Patients may variously feel a tingling or stinging numbness or pain in the toes or fingers; an electric, shooting pain; loss of sense of touch; loss of heat/cold sensation; loss of power in the arms/legs; difficulty in grasping objects; or they may fall when walking. There are few effective remedies once peripheral neuropathy appears as a result of chemotherapy. In some cases the neuropathy may be almost irreversible. If symptoms are severe, the anticancer drug treatment must be discontinued or the prescription should be changed. In most cases, neuropathy persists as long as chemotherapy continues, and the symptoms do not disappear completely even after treatment ends, and complete recovery may take a long time. Treatment for peripheral neuropathy is not yet well established. Common medications including the combined use of calcium and magnesium or vitamin B6 and B12 have been reported to be effective to relieve numbness. The main symptomatic treatments for neuropathic pain include antidepressants, NSAIDs, or serotonin and norepinephrine reuptake inhibitors. If pain is severe, morphine and other narcotic analgesics may also be prescribed [56].

2.3. Indications and Evidence for Kampo Therapy for Chemotherapy-Induced Peripheral Neuropathy. The use of the Kampo preparation goshajinkigan [57-67] as drug therapy for peripheral neuropathy symptoms has been widely reported in Japan. Goshajinkigan extract preparation has been reported to relieve symptoms such as numbness or pain in $80 \%$ of cases in which it is used for peripheral neuropathy caused by paclitaxel for breast cancer [68]. Goshajinkigan also improves subjective symptoms of peripheral neuropathy due to the combined use of paclitaxel and carboplatin for ovarian or uterine cancers. Neuropathy is a characteristic adverse effect of oxaliplatin, the core drug for colorectal cancer. A high incidence of symptoms such as extremity numbness and cold sensation has been observed with the continued therapeutic use of oxaliplatin, especially at a cumulative dose over $500 \mathrm{mg} / \mathrm{m}^{2}$. Treatment can be continued if symptoms are mild, but the dosage is decreased or the administration is discontinued in some severe cases. On the other hand, research has found that goshajinkigan can alleviate such symptoms. Nishioka et al. [69] and Kono et al. [70] conducted a retrospective comparison and examination of the effects of goshajinkigan for peripheral neuropathy associated with oxaliplatin in advanced or recurrent colorectal cancer patients. They found that the group which was administered goshajinkigan from the start of chemotherapy tolerated the largest dosage until onset of peripheral neuropathy. Goshajinkigan's efficacy differs according to the causal anticancer drug. It promises some effectiveness for numbness caused
TABLE 1: Goshajinkigan extract granules for ethical use.

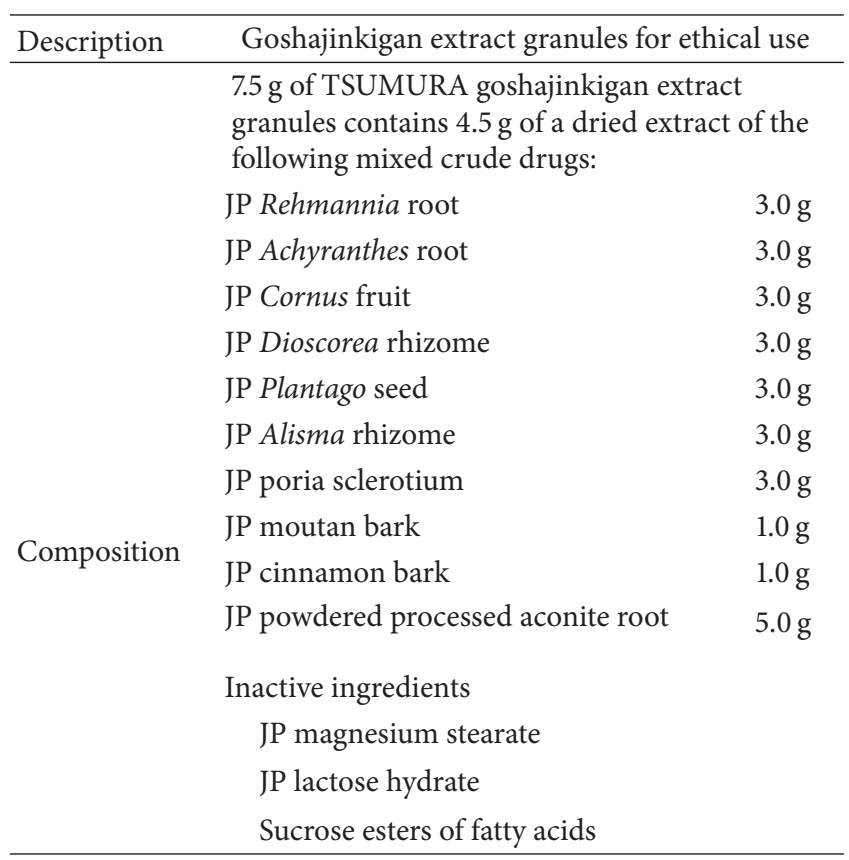

(JP: The Japanese Pharmacopoeia.)

by paclitaxel, and so forth, but it is virtually ineffective for oxaliplatin. Since it might be effective for prevention of oxaliplatin-induced neuropathy, it would be better to administer goshajinkigan from the start of chemotherapy. It has been reported that administration of Kampo preparations promises an increase in the frequency of administration during the FOLFOX regimen, which centers on oxaliplatin, before onset of numbness as an adverse effect $[58,70]$.

2.4. Goshajinkigan. Goshajinkigan's Kampo constituents and HPLC fingerprint appear in Table 1 and Figure 1.

Goshajinkigan is indicated for the relief of the following symptoms in patients with decreased urine volume or polyuria, occasional dry mouth, proneness to fatigue, and sensitivity to cold in the extremities: leg pain, low back pain, numbness, blurred vision (elderly), pruritus, dysuria, and edema. Goshajinkigan consists of 10 constituent crude drugs (Table 1) and is a prescription with fortified effectiveness against swelling, numbness, and arthralgia, in addition to the beneficial effects of hachimijiogan. Specifically, goshajinkigan is a Kampo preparation that improves blood circulation, has a body warming analgesic action, and reduces swelling. It is used for patients with remarkable edema tendency, severe arthralgia, and persistent low back pain. It is frequently used for symptoms in which peripheral vascular disease is suspected of being involved, such as sciatica and diabetic neuropathy, and has demonstrated effectiveness for these conditions. The usefulness of goshajinkigan is conjectured to be aconitine [71]. Shakuyakukanzoto is a Kampo preparation used for various types of myalgia including menstrual pain and cramp [72]. Shakuyakukanzoto has been reported to demonstrate effectiveness for arthralgia and 


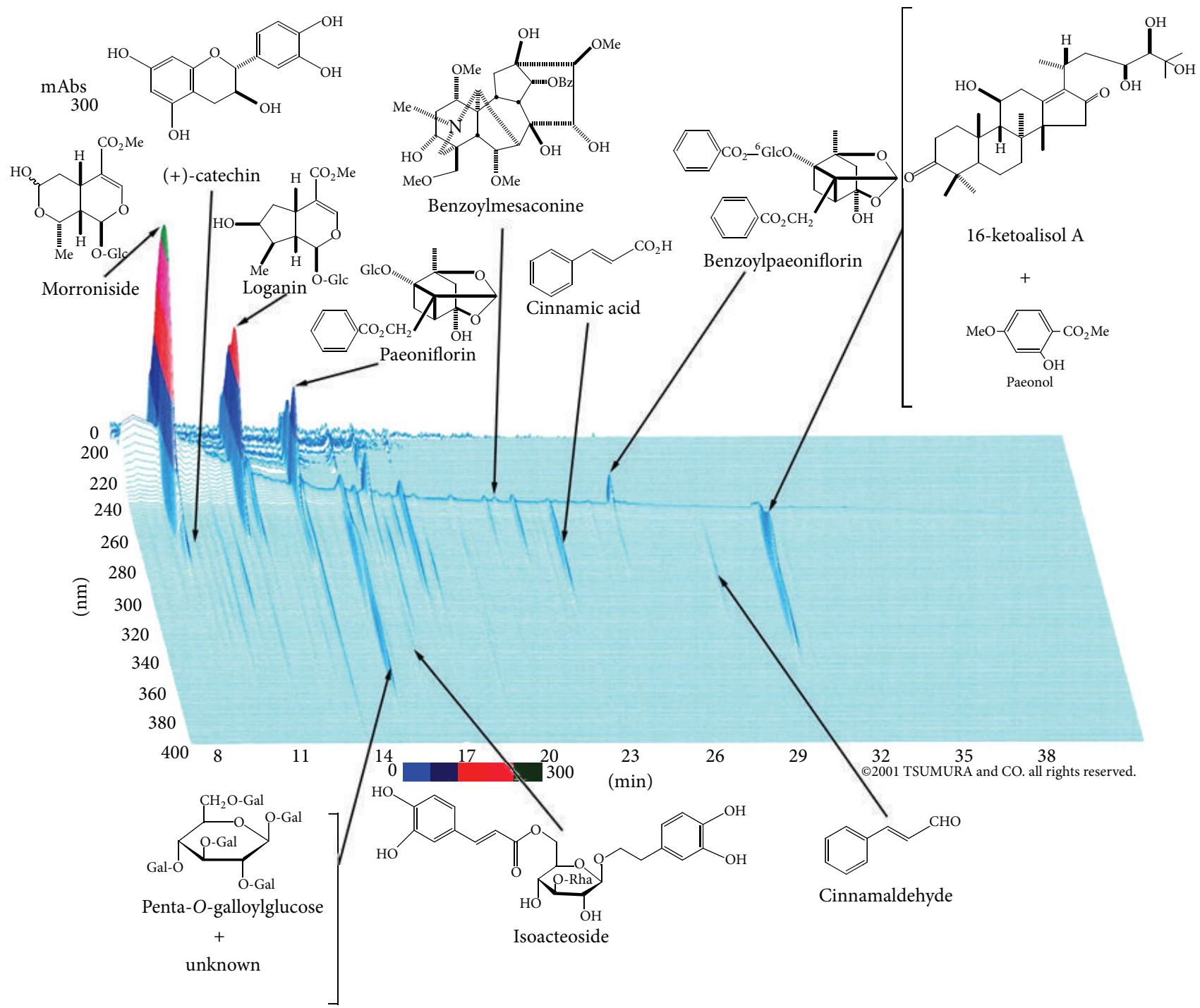

FIGURE 1: 3D-HPLC pattern of TJ-107 goshajinkigan (this 3D-HPLC was created in 2001 by TSUMURA and CO.).

myalgia due to paclitaxel [73]. While it is impossible to completely control peripheral neuropathy and myalgia caused by anticancer drugs, the combined use of goshajinkigan and shakuyakukanzoto may enhance improvement of subjective symptoms.

\section{Kampo for Chemotherapy-Induced Diarrhea}

3.1. Development of a Camptothecin Derivative-Irinotecan. Irinotecan is an anticancer drug classified as a plant alkaloid. It inhibits cancer cell proliferation by breaking DNA during cell division through its inhibition of the enzyme topoisomerase, which is required when DNA replicates. Wall et al. extracted and isolated camptothecin (CPT) in 1966 from Camptotheca acuminate, a plant native to China, and found that it has a powerful antineoplastic effect [74]. Subsequent development was undertaken by the National Cancer Institute (NCI) in the USA but was abandoned following the emergence of adverse effects. Pharmaceutical manufacturers in Japan vigorously pursued synthetic research into derivatives to preserve CPT's activity while reducing its toxicity, resulting in the CPT derivative irinotecan, which has been subsequently used as a potent anticancer drug. Irinotecan has demonstrated usefulness for various types of cancer, including colon and lung cancers, and its applications have been widening. Irinotecan suppresses the action of topoisomerase 1, which is involved in DNA replication, thereby demonstrating a strong antitumor effect; however, it can cause severe adverse effects including leukopenia and diarrhea $[16,17,75-94]$.

3.2. Adverse Effects of Irinotecan and Their Frequency. The chief adverse effects are severe myelosuppression and intractable diarrhea. There have been reports of death following severe infection due to myelosuppression, intractable diarrhea, and intestinal perforation due to intestinal paralysis or bowel obstruction. Irinotecan undergoes metabolism 
in the liver where it is converted into the active metabolite SN-38, setting off an antitumor action. SN-38 is then deactivated by conjugation reaction by uridine diphosphate glucuronosyltransferase (UGT) and excreted into the duodenum through the biliary tract. However, individual variability in the UGT activity is thought to be a reason for individual variation in the adverse effects of irinotecan. Many reports [95-99] in recent years mention the relation between UGT1A1 genetic polymorphism and onset of adverse effects of irinotecan. UGT1A1 is a molecular species of UGT in the liver and is the enzyme that metabolizes irinotecan. $\mathrm{UGT1A1}^{*} 28$ and ${ }^{*} 6$ are variants of UGT1A1, and reports cite an increase in the incidence of severe adverse effects of irinotecan due to reduced UGT1A1 activity.

The most troublesome adverse effect of CPT-11 is severe delayed diarrhea, which is caused by reactivation in the digestive tract by enteric bacterial $\beta$-glucuronidase. CPT-11 is a prodrug firstly decomposed by carboxyl esterase in the liver into SN-38, which has a powerful anticancer action and is then transported throughout the body. The SN-38 formed in the liver is glucuronidated by a glucuronidation enzyme also present in the liver. At this point, the SN-38 is deactivated, losing its injurious effect. However, after being excreted into the digestive tract via the biliary tract, the SN-38 is decomposed by enteric bacterial $\beta$-glucuronidase, thereby reforming SN-38. It is surmised that this SN-38 formed in the digestive tract then damages intestinal mucosal epithelial cells, giving rise to delayed diarrhea.

3.3. Irinotecan Hydrochloride-Induced Diarrhea and Kampo. The flavonoid glycoside baicalin may control irinotecan hydrochloride-induced diarrhea because it actively inhibits $\beta$-glucuronidase of intestinal flora and suppresses reformation of the active form (SN-38) in the digestive tract. Large amounts of baicalin are contained in Scutellaria root, a constituent crude drug of Kampo preparations. Researchers have tested hangeshashinto for diarrhea as it is a Kampo preparation containing Scutellaria root. It has been reported in human clinical trials and animal experiments that administration of hangeshashinto [37, 100-104] extract formulation two to three days before irinotecan hydrochloride administration effectively prevents or reduces diarrhea. It has been ascertained that this does not affect the antitumor action.

If the preventative effect against irinotecan hydrochloride-induced diarrhea is contained solely in the action mechanism of the flavonoid glycoside-induced $\beta$-glucuronidase inhibition, single administration of a flavonoid glycoside or Scutellaria root, rather than a Kampo formulation, may also be effective. Nevertheless, the comprehensive actions of the other crude drugs contained in hangeshashinto improve effectiveness. Specifically, it has been reported that hangeshashinto suppresses elevation of enteric prostaglandin E2, promotes repair of damaged intestinal mucosa, and improves intestinal water absorption $[37,100]$. A particular characteristic of Kampo preparations is that they give greater efficacy through the synergistic effect of multiple crude drugs compared to one constituent alone. Loperamide hydrochloride is often administered for irinotecan hydrochloride-induced diarrhea, yet in some cases it is ineffective, maybe because
TABLE 2: Hangeshashinto extract granules for ethical use.

\begin{tabular}{|c|c|c|}
\hline Description & \multicolumn{2}{|c|}{ Hangeshashinto extract granules for ethical use } \\
\hline & \multicolumn{2}{|c|}{$\begin{array}{l}7.5 \mathrm{~g} \text { of TSUMURA hangeshashinto extract } \\
\text { granules contains } 4.5 \mathrm{~g} \text { of a dried extract of the } \\
\text { following mixed crude drugs: }\end{array}$} \\
\hline & JP Pinellia tuber & $5.0 \mathrm{~g}$ \\
\hline & JP Scutellaria root & $2.5 \mathrm{~g}$ \\
\hline & JP processed ginger & $2.5 \mathrm{~g}$ \\
\hline & JP Glycyrrhiza & $2.5 \mathrm{~g}$ \\
\hline & JP jujube & $2.5 \mathrm{~g}$ \\
\hline \multirow[t]{6}{*}{ Composition } & JP Ginseng & $2.5 \mathrm{~g}$ \\
\hline & JP Coptis rhizome & $1.0 \mathrm{~g}$ \\
\hline & \multicolumn{2}{|l|}{ Inactive ingredients } \\
\hline & \multicolumn{2}{|c|}{ JP magnesium stearate } \\
\hline & \multicolumn{2}{|c|}{ JP lactose hydrate } \\
\hline & \multicolumn{2}{|c|}{ Sucrose esters of fatty acids } \\
\hline
\end{tabular}

(JP: The Japanese Pharmacopoeia.)

loperamide does not cure intestinal mucosal damage. The Tochigi Cancer Center Research Group published the results of a clinical study that compared the degree of diarrhea in 41 patients with advanced non-small-cell lung cancer following anticancer drug treatment with irinotecan hydrochloride and cisplatin. Eighteen patients were administered hangeshashinto while the control group (without hangeshashinto administration) consisted of 23 patients. No significant difference in diarrhea frequency or interval was observed between the hangeshashinto group and the nonadministration group; however, the frequency of severe grade three and four diarrheas was lower in the hangeshashinto group [37].

\subsection{Hangeshashinto. The Kampo constituents and HPLC fin-} gerprint appear in Table 2 and Figure 2.

Hangeshashinto is indicated for the relief of the following symptoms in those patients with blocked feeling in the stomach pit and occasional nausea, vomiting, anorexia, borborygmus, and a tendency of loose stool or diarrhea. The targeted diseases and symptoms are as follows: acute or chronic gastrointestinal catarrh, fermentative diarrhea, dyspepsia, gastroptosis, nervous gastritis, gastrasthenia, hangover, belching, heartburn, stomatitis, and neurosis.

The reference sources for hangeshashinto are "Shokanron" and "Kinki-yoryaku". Hangeshashinto consists of seven crude drugs. Pinellia Tuber clears fluid retention in the stomach and stops vomiting, while together with Coptis rhizome and Scutellaria root, it clears gastrointestinal inflammation. Coptis rhizome and Scutellaria root are bitter, and they are good for the stomach and have anti-inflammatory actions. Ginseng and ginger improve gastrointestinal blood flow and promote the recovery of gastrointestinal function. Glycyrrhiza and jujube harmonize crude drugs and enhance their cooperative effects. 


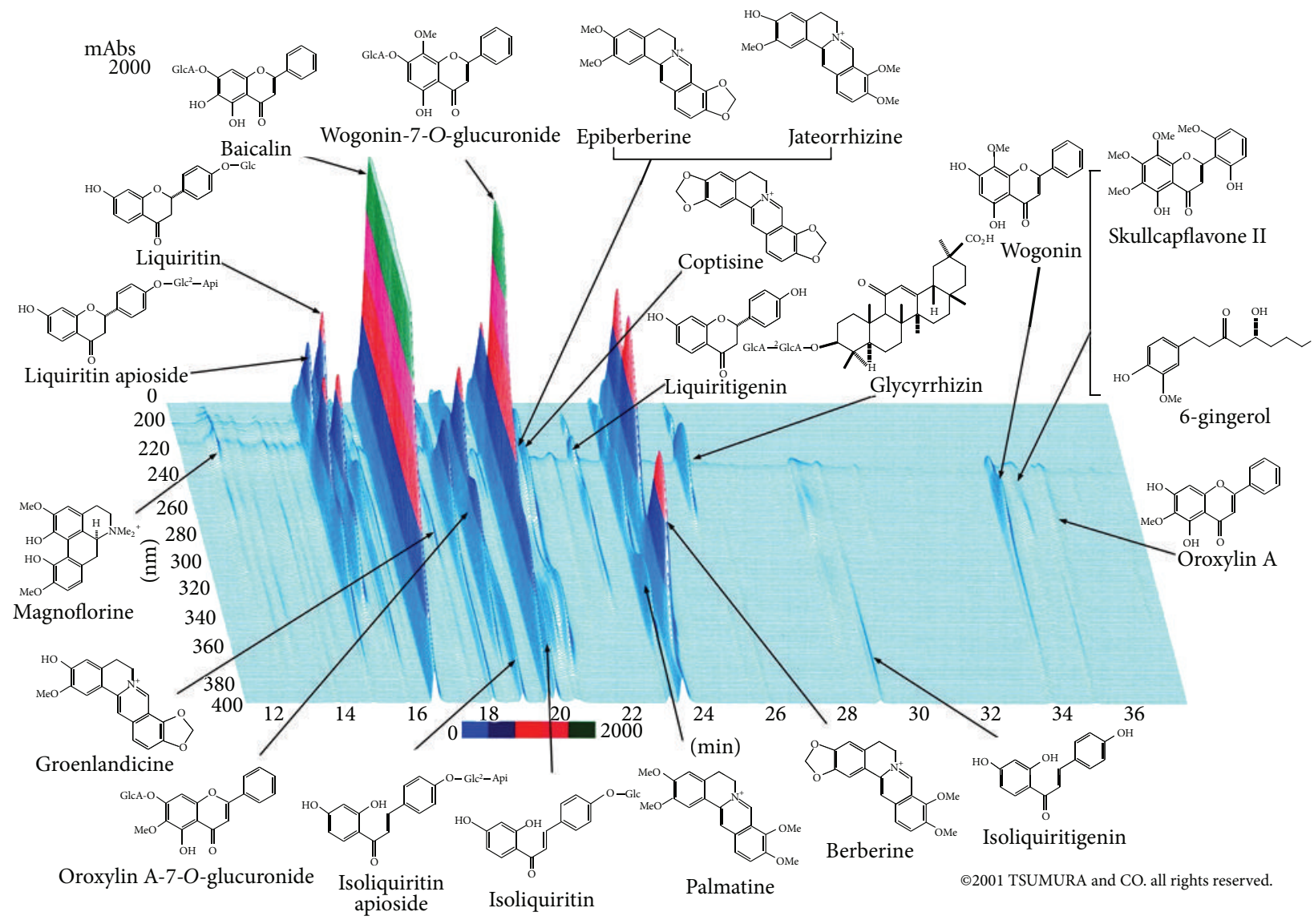

FIgURE 2: 3D-HPLC pattern of TJ-14 hangeshashinto (this 3D-HPLC was created in 2001 by TSUMURA and CO.).

\section{Conclusions}

Steady progress is being made in the treatment of cancer. However, highly invasive treatment can cause patients' distress. It would seem to be a natural progress that traditional, complementary, and alternative forms of medical care are now being adopted to alleviate the attendant suffering. Some express opposition to the combined use of Kampo preparations with anticancer drugs or surgery. Yet, if appropriate Kampo preparations alleviate adverse effects of cancer treatment, improve QOL, enhance therapeutic efficacy, and prolong life, the importance of treatment that includes Kampo preparations as palliative or supportive care for cancer will go on growing. Western and Kampo medicines coexist in Japan as a characteristic form of medical care. This combination needs to be further promoted and also to be established as integrative medicine based on scientific evidence.

\section{Acknowledgments}

This work was supported in part by the Grants-in-Aid for Scientific Research by Grant for Assist KAKEN from Kanazawa Medical University (K2012-8) and Japan Society for the Promotion of Science (JSPS) no. 22590669.

\section{References}

[1] F. Ferrara and C. A. Schiffer, "Acute myeloid leukaemia in adults," The Lancet, vol. 381, pp. 484-495, 2013.

[2] A. Pennathur, M. K. Gibson, B. A. Jobe et al., "Oesophageal carcinoma," The Lancet, vol. 381, pp. 400-412, 2013.

[3] T. Waddell and D. Cunningham, "Impact of targeted neoadjuvant therapies in the treatment of solid organ tumours," British Journal of Surgery, vol. 100, pp. 5-14, 2013.

[4] T. C. Chua and A. Saxena, "Preoperative chemoradiation followed by surgical resection for resectable pancreatic cancer: a review of current results," Surgical Oncology, vol. 20, no. 4, pp. e161-e168, 2011.

[5] L. T. MacEdo, J. Ribeiro, G. Curigliano et al., "Multidisciplinary approach in the treatment of patients with small cell bladder carcinoma," European Journal of Surgical Oncology, vol. 37, no. 7, pp. 558-562, 2011.

[6] J. J. Liu, J. Lu, and M. J. McKeage, "Membrane transporters as determinants of the pharmacology of platinum anticancer drugs," Current Cancer Drug Targets, vol. 12, pp. 962-986, 2012.

[7] X. Song, S. R. Long, B. Barber et al., "Systematic review on infusion reactions associated with chemotherapies and monoclonal antibodies for metastatic colorectal cancer," Current Clinical Pharmacology, vol. 7, no. 1, pp. 56-65, 2012. 
[8] M. J. Deenen, A. Cats, J. H. Beijnen, and J. H. M. Schellens, "Part 2: pharmacogenetic variability in drug transport and phase I anticancer drug metabolism," Oncologist, vol. 16, no. 6, pp. 820$834,2011$.

[9] E. S. Hsu, "A review of granisetron, 5-hydroxytryptamine3 receptor antagonists, and other antiemetics," American Journal of Therapeutics, vol. 17, no. 5, pp. 476-486, 2010.

[10] C. Lee, M. Gianos, and W. B. Klaustermeyer, "Diagnosis and management of hypersensitivity reactions related to common cancer chemotherapy agents," Annals of Allergy, Asthma and Immunology, vol. 102, no. 3, pp. 179-187, 2009.

[11] J. Bardy, N. J. Slevin, K. L. Mais, and A. Molassiotis, "A systematic review of honey uses and its potential value within oncology care," Journal of Clinical Nursing, vol. 17, no. 19, pp. 2604-2623, 2008.

[12] C. Grande, M. J. Villanueva, G. Huidobro, and J. Casal, "Docetaxel-induced interstitial pneumonitis following nonsmall-cell lung cancer treatment," Clinical and Translational Oncology, vol. 9, no. 9, pp. 578-581, 2007.

[13] E. A. Rodriguez-Frias and W. M. Lee, "Cancer chemotherapy II: atypical hepatic injuries," Clinics in Liver Disease, vol. 11, no. 3, pp. 663-676, 2007.

[14] E. A. Rodriguez-Frias and W. M. Lee, "Cancer chemotherapy I: hepatocellular injury," Clinics in Liver Disease, vol. 11, no. 3, pp. 641-662, 2007.

[15] T. M. Bosch, I. Meijerman, J. H. Beijnen, and J. H. M. Schellens, "Genetic polymorphisms of drug-metabolising enzymes and drug transporters in the chemotherapeutic treatment of cancer," Clinical Pharmacokinetics, vol. 45, no. 3, pp. 253-285, 2006.

[16] M. Ando, Y. Hasegawa, and Y. Ando, "Pharmacogenetics of irinotecan: a promoter polymorphism of UGT1A1 gene and severe adverse reactions to irinotecan," Investigational New Drugs, vol. 23, no. 6, pp. 539-545, 2005.

[17] Y. Ando and Y. Hasegawa, "Clinical pharmacogenetics of irinotecan (CPT-11)," Drug Metabolism Reviews, vol. 37, no. 3, pp. 565-574, 2005.

[18] R.-J. Bensadoun, N. Magné, P.-Y. Marcy, and F. Demard, "Chemotherapy- and radiotherapy-induced mucositis in head and neck cancer patients: new trends in pathophysiology, prevention and treatment," European Archives of Oto-RhinoLaryngology, vol. 258, no. 9, pp. 481-487, 2001.

[19] R. L. Nahin, "Use of the best case series to evaluate complementary and alternative therapies for cancer: a systematic review," Seminars in Oncology, vol. 29, no. 6, pp. 552-562, 2002.

[20] J. D. White, "Complementary and alternative medicine research: a National Cancer Institute perspective," Seminars in Oncology, vol. 29, no. 6, pp. 546-551, 2002.

[21] M. A. Richardson and S. E. Straus, "Complementary and alternative medicine: opportunities and challenges for cancer management and research," Seminars in Oncology, vol. 29, no. 6, pp. 531-545, 2002.

[22] J. D. White, “The National Cancer Institute's perspective and agenda for promoting awareness and research on alternative therapies for cancer," Journal of Alternative and Complementary Medicine, vol. 8, no. 5, pp. 545-550, 2002.

[23] L. A. Walker and S. Budd, "UK: the current state of regulation of complementary and alternative medicine," Complementary Therapies in Medicine, vol. 10, no. 1, pp. 8-13, 2002.

[24] J. D. White, "Cancer: current research in alternative therapies," Primary Care, vol. 29, pp. 379-392, 2002.
[25] M. Markman, "Safety issues in using complementary and alternative medicine," Journal of Clinical Oncology, vol. 20, no. 18 , pp. 39-41, 2002.

[26] T. Tsutsui and N. Muramatsu, "Japan's universal long-term care system reform of 2005: containing costs and realizing a vision," Journal of the American Geriatrics Society, vol. 55, no. 9, pp. 1458-1463, 2007.

[27] N. Ikegami, "The economics of health care in Japan," Science, vol. 258, pp. 614-618, 1992.

[28] K. Terasawa, "Evidence-based reconstruction of Kampo medicine: part I-is Kampo CAM?" Evidence-Based Complementary and Alternative Medicine, vol. 1, pp. 11-16, 2004.

[29] K. Terasawa, "Evidence-based reconstruction of Kampo medicine: part II-the concept of Sho," Evidence-Based Complementary and Alternative Medicine, vol. 1, pp. 119123, 2004.

[30] K. Terasawa, "Evidence-based reconstruction of Kampo medicine: part-III-how should Kampo be evaluated?" Evidence-Based Complementary and Alternative Medicine, vol. 1, pp. 219-222, 2004.

[31] Y. Motoo, T. Seki, and K. Tsutani, "Traditional Japanese medicine, Kampo: its history and current status," Chinese Journal of Integrative Medicine, vol. 17, no. 2, pp. 85-87, 2011.

[32] S. Iwase, T. Yamaguchi, T. Miyaji et al., "The clinical use of Kampo medicines (traditional Japanese herbal treatments) for controlling cancer patients' symptoms in Japan: a national cross-sectional survey," BMC Complementary and Alternative Medicine, vol. 20, article 222, 2012.

[33] K. Ogawa, T. Omatsu, C. Matsumoto et al., "Protective effect of the Japanese traditional medicine juzentaihoto on myelosuppression induced by the anticancer drug TS-1 and identification of a potential biomarker of this effect," BMC Complementary and Alternative Medicine, vol. 9, article 118, 2012.

[34] J. J. Gao, P. P. Song, F. H. Qi et al., "Evidence-based research on traditional Japanese medicine, Kampo, in treatment of gastrointestinal cancer in Japan," Drug Discoveries \& Therapeutics, vol. 6, pp. 1-8, 2012.

[35] H. Yasunaga, H. Miyata, H. Horiguchi, K. Kuwabara, H. Hashimoto, and S. Matsuda, "Effect of the Japanese herbal kampo medicine Dai-kenchu-to on postoperative adhesive small bowel obstruction requiring long-tube decompression: a propensity score analysis," Evidence-based Complementary and Alternative Medicine, vol. 2011, Article ID 264289, 7 pages, 2011.

[36] R. Sharma, P. Tobin, and S. J. Clarke, "Management of chemotherapy-induced nausea, vomiting, oral mucositis, and diarrhoea," The Lancet Oncology, vol. 6, no. 2, pp. 93-102, 2005.

[37] K. Mori, T. Kondo, Y. Kamiyama, Y. Kano, and K. Tominaga, "Preventive effect of Kampo medicine (Hangeshashinto) against irinotecan-induced diarrhea in advanced non-smallcell lung cancer," Cancer Chemotherapy and Pharmacology, vol. 51, no. 5, pp. 403-406, 2003.

[38] W. T. Lim, E. H. Tan, C. K. Toh et al., "Phase I pharmacokinetic study of a weekly liposomal paclitaxel formulation (GenexolPM) in patients with solid tumors," Annals of Oncology, vol. 21, no. 2, Article ID mdp315, pp. 382-388, 2010.

[39] M. N. Fornier, "Epothilones in breast cancer: review of clinical experience," Annals of Oncology, vol. 18, no. 5, pp. v16-v21, 2007.

[40] F. Hilpert, A. Stähle, O. Tomé et al., "Neuroprotection with amifostine in the first-line treatment of advanced ovarian cancer with carboplatin/paclitaxel-based chemotherapy-a doubleblind, placebo-controlled, randomized phase II study from 
the Arbeitsgemeinschaft Gynäkologische Onkologoie (AGO) Ovarian Cancer Study Group," Supportive Care in Cancer, vol. 13, no. 10, pp. 797-805, 2005.

[41] M. J. Edelman, "Gemcitabine and carboplatin regimens in advanced non-small-cell lung cancer: focus on randomized phase III trials," Clinical Lung Cancer, vol. 4, pp. 40-44, 2003.

[42] A. D. Seidman, J. O’Shaughnessy, and J.-L. Misset, "Singleagent capecitabine: a reference treatment for taxane-pretreated metastatic breast cancer?" Oncologist, vol. 7, no. 6, pp. 20-28, 2002.

[43] C. Nicolaides, M. A. Dimopoulos, E. Samantas et al., "Gemcitabine and vinorelbine as second-line treatment in patients with metastatic breast cancer progressing after first-line taxanebased chemotherapy: a phase II study conducted by the Hellenic Cooperative Oncology Group," Annals of Oncology, vol. 11, no. 7, pp. 873-875, 2000.

[44] N. Ami, K. Okamoto, and H. Oshima, "Analgesic effect of magnetic stimulation on paclitaxel-induced peripheral neuropathic pain in mice," Brain Research, vol. 21, pp. 24-29, 2012.

[45] C. Tofthagen, J. Overcash, and K. Kip, "Falls in persons with chemotherapy-induced peripheral neuropathy," Supportive Care in Cancer, vol. 20, no. 3, pp. 583-589, 2012.

[46] M. H. Cohen, P. Cortazar, R. Justice, and R. Pazdur, "Approval summary: pemetrexed maintenance therapy of advanced/metastatic nonsquamous, non-small cell lung cancer (NSCLC)," Oncologist, vol. 15, no. 12, pp. 1352-1358, 2010.

[47] C. Tofthagen, "Patient perceptions associated with chemotherapy-induced peripheral neuropathy," Clinical Journal of Oncology Nursing, vol. 14, no. 3, pp. E22-E28, 2010.

[48] E. K. Joseph and J. D. Levine, "Comparison of oxaliplatinand cisplatin-induced painful peripheral neuropathy in the rat," Journal of Pain, vol. 10, no. 5, pp. 534-541, 2009.

[49] S. R. McWhinney, R. M. Goldberg, and H. L. McLeod, "Platinum neurotoxicity pharmacogenetics," Molecular Cancer Therapeutics, vol. 8, no. 1, pp. 10-16, 2009.

[50] F. V. Piantedosi, F. Caputo, G. Mazzarella et al., "Gemcitabine, ifosfamide and paclitaxel in advanced/metastatic non-small cell lung cancer patients: a phase II study," Cancer Chemotherapy and Pharmacology, vol. 61, no. 5, pp. 803-807, 2008.

[51] Y.-M. Chen, R.-P. Perng, J.-F. Shih, C.-M. Tsai, and J. WhangPeng, "Chemotherapy for non-small cell lung cancer in elderly patients," Chest, vol. 128, no. 1, pp. 132-139, 2005.

[52] D. A. Anthoney, M. J. McKean, J. T. Roberts et al., "Bleomycin, vincristine, cisplatin/bleomycin, etoposide, cisplatin chemotherapy: an alternating, dose intense regimen producing promising results in untreated patients with intermediate or poor prognosis malignant germ-cell tumours," British Journal of Cancer, vol. 90, no. 3, pp. 601-606, 2004.

[53] G. Oliverio, D. Canuti, A. Tononi et al., "Paclitaxel efficacy and tolerability in second-line treatment of refractory and relapsed ovarian cancer patients," Journal of Chemotherapy, vol. 11, no. 4, pp. 301-305, 1999.

[54] J. P. Guastalla, E. Pujade-Lauraine, B. Weber et al., "Efficacy and safety of the paclitaxel and carboplatin combination in patients with previously treated advanced ovarian carcinoma. A multicenter GINECO (Group d'Investigateurs Nationaux pour l'Etude des Cancers Ovariens) phase II study," Annals of Oncology, vol. 9, pp. 37-43, 1998.

[55] L. M. Schuchter, "Exploration of platinum-based dose-intensive chemotherapy strategies with amifostine (Ethyol@)," European Journal of Cancer Part A, vol. 32, supplement 4, pp. S40-S42, 1996.
[56] W. Grisold, G. Cavaletti, and A. J. Windebank, "Peripheral neuropathies from chemotherapeutics and targeted agents: diagnosis, treatment, and prevention," Journal of Neuro-Oncology, vol. 4, pp. 45-54, 2012.

[57] S. Ushio, N. Egashira, H. Sada et al., "Goshajinkigan reduces oxaliplatin-induced peripheral neuropathy without affecting anti-tumour efficacy in rodents," European Journal of Cancer, vol. 48, pp. 1407-1413, 2012.

[58] M. Nishioka, M. Shimada, N. Kurita et al., "The Kampo medicine, Goshajinkigan, prevents neuropathy in patients treated by FOLFOX regimen," International Journal of Clinical Oncology, vol. 16, no. 4, pp. 322-327, 2011.

[59] X. Hu, J. Sato, G. Bajotto et al., "Goshajinkigan (Chinese herbal medicine niu-che-sen-qi-wan) improves insulin resistance in diabetic rats via the nitric oxide pathway," Nagoya Journal of Medical Science, vol. 72, no. 1-2, pp. 35-42, 2010.

[60] T. Kono, N. Mamiya, N. Chisato et al., "Efficacy of goshajinkigan for peripheral neurotoxicity of oxaliplatin in patients with advanced or recurrent colorectal cancer," Evidence-based Complementary and Alternative Medicine, vol. 2011, Article ID 418481, 7 pages, 2011.

[61] B. Qin, M. Nagasaki, M. Ren et al., "Gosha-jinki-gan (a Herbal Complex) corrects abnormal insulin signaling," Evidence-Based Complementary and Alternative Medicine, vol. 1, pp. 269-276, 2004.

[62] Y. Nagaki, S. Hayasaka, Y. Hayasaka et al., "Effects of Goshajinkigan on corneal sensitivity, superficial punctate keratopathy and tear secretion in patients with insulin-dependent diabetes mellitus," American Journal of Chinese Medicine, vol. 31, no. 1, pp. 103-109, 2003.

[63] X. Hu, J. Sato, Y. Oshida, M. Xu, G. Bajotto, and Y. Sato, "Effect of Gosha-jinki-gan (Chinese herbal medicine: Niu-Che-Sen-QiWan) on insulin resistance in streptozotocin-induced diabetic rats," Diabetes Research and Clinical Practice, vol. 59, no. 2, pp. 103-111, 2003.

[64] Y. Suzuki, K. Goto, A. Ishige, Y. Komatsu, and J. Kamei, "Antinociceptive effect of Gosha-jinki-gan, a Kampo medicine, in streptozotocin-induced diabetic mice," Japanese Journal of Pharmacology, vol. 79, no. 2, pp. 169-175, 1999.

[65] M. Nishizawa, W. H. F. Sutherland, and H. Nukada, "Goshajinki-gan (herbal medicine) in streptozocin-induced diabetic neuropathy," Journal of the Neurological Sciences, vol. 132, no. 2, pp. 177-181, 1995.

[66] M. Tawata, A. Kurihara, K. Nitta, E. Iwase, N. Gan, and T. Onaya, "The effects of Goshajinkigan, a herbal medicine, on subjective symptoms and vibratory threshold in patients with diabetic neuropathy," Diabetes Research and Clinical Practice, vol. 26, no. 2, pp. 121-128, 1994.

[67] T. Takashima, "Application of Goshajinkigan to the peripheral neuropathy of paclitaxel in a breast cancer," Clinical Cancer Research, vol. 51, pp. 58-59, 2005.

[68] T. Yamamoto, T. Murai, M. Ueda et al., "Clinical features of paclitaxel-induced peripheral neuropathy and role of Gosyajinki-gan," Gan To Kagaku Ryoho, vol. 36, no. 1, pp. 89-92, 2009.

[69] M. Nishioka, M. Shimada, N. Kurita et al., "The Kampo medicine, Goshajinkigan, prevents neuropathy in patients treated by FOLFOX regimen," International Journal of Clinical Oncology, vol. 16, no. 4, pp. 322-327, 2011.

[70] T. Kono, N. Mamiya, N. Chisato et al., "Efficacy of goshajinkigan for peripheral neurotoxicity of oxaliplatin in patients with advanced or recurrent colorectal cancer," Evidence-Based 
Complementary and Alternative Medicine, vol. 2011, Article ID 418481, 8 pages, 2011.

[71] T. Oyama, T. Isono, Y. Suzuki, and Y. Hayakawa, "Antinociceptive effects of aconiti tuber and its alkaloids," American Journal of Chinese Medicine, vol. 22, no. 2, pp. 175-182, 1994.

[72] K. Yamamoto, H. Hoshiai, and K. Noda, "Effects of Shakuyakukanzo-to on muscle pain from combination chemotherapy with paclitaxel and carboplatin," Gynecologic Oncology, vol. 81, no. 2, pp. 333-334, 2001.

[73] K. Yamamoto, T. Abe, K. Fujii et al., "Examination about a cancer chemotherapy enforcement case including the usefulness Paclitaxel of the Chinese medicine to numbness and muscular pain," Obstetrics and Gynecology in Chinese Medicine, vol. 17, pp. 24-27, 2000.

[74] M. E. Wall, M. C. Wani, C. E. Cook, K. H. Palmer, A. T. McPhail, and G. A. Sim, "Plant antitumor agents. I. The isolation and structure of camptothecin, a novel alkaloidal leukemia and tumor inhibitor from Camptotheca acuminata," Journal of the American Chemical Society, vol. 88, no. 16, pp. 3888-3890, 1966.

[75] G. G. Chabot, "Clinical pharmacokinetics of irinotecan," Clinical Pharmacokinetics, vol. 33, no. 4, pp. 245-259, 1997.

[76] S. Gail Eckhardt, "Irinotecan: a review of the initial phase I trials," Oncology, vol. 12, no. 8, pp. 31-38, 1998.

[77] J. G. Kuhn, "Pharmacology of irinotecan," Oncology, vol. 12, pp. 39-42, 1998.

[78] J. Randolph Hecht, "Gastrointestinal toxicity of Irinotecan," Oncology, vol. 12, no. 8, pp. 72-78, 1998.

[79] S. Wadler, A. B. Benson III, C. Engelking et al., "Recommended guidelines for the treatment of chemotherapy-induced diarrhea," Journal of Clinical Oncology, vol. 16, no. 9, pp. 3169-3178, 1998.

[80] Y. Sugiyama, Y. Kato, and X.-Y. Chu, "Multiplicity of biliary excretion mechanisms for the camptothecin derivative irinotecan (CPT-11), its metabolite SN-38, and its glucuronide: role of canalicular multispecific organic anion transporter and Pglycoprotein," Cancer Chemotherapy and Pharmacology, vol. 42, no. 1, supplement, pp. S44-S49, 1998.

[81] R. J. Cersosimo, "Irinotecan: a new antineoplastic agent for the management of colorectal cancer," Annals of Pharmacotherapy, vol. 32, no. 12, pp. 1324-1333, 1998.

[82] M. Ogawa, "Novel anticancer drugs in Japan," Journal of Cancer Research and Clinical Oncology, vol. 125, no. 3-4, pp. 134-140, 1999.

[83] H. Bleiberg, “CPT-11 in gastrointestinal cancer," European Journal of Cancer, vol. 35, no. 3, pp. 371-379, 1999.

[84] M. Fukuoka, N. Masuda, S. Kudoh, and S. Negoro, "Irinotecan in small-cell lung cancer-Japanese trials," Oncology, vol. 14, no. 7, pp. 57-62, 2000.

[85] Y. Ando, H. Saka, M. Ando et al., "Polymorphisms of UDPglucuronosyltransferase gene and irinotecan toxicity: a pharmacogenetic analysis," Cancer Research, vol. 60, no. 24, pp. 6921-6926, 2000.

[86] J. R. Murren, K. Blum, M. Gallipoli, A. Mckeon, and R. Rich, "Rationale and dose-finding studies of the combination of irinotecan and a taxane on a weekly schedule," Oncology, vol. 15, no. 1, pp. 25-30, 2001.

[87] A. Font, J. M. Sanchez, R. Rosell et al., "Phase I study of weekly CPT-11 (irinotecan)/docetaxel in patients with advanced solid tumors," Lung Cancer, vol. 37, no. 2, pp. 213-218, 2002.

[88] H. Fujii, M. Koshiyama, M. Konishi, M. Yoshida, and K. Tauchi, "Intermittent, repetitive administrations of irinotecan (CPT- 11) reduces its side-effects," Cancer Detection and Prevention, vol. 26, no. 3, pp. 210-212, 2002.

[89] G. Toffoli, E. Cecchin, G. Corona, and M. Boiocchi, "Pharmacogenetics of irinotecan," Current Medicinal Chemistry, vol. 3, no. 3, pp. 225-237, 2003.

[90] A. B. Benson III, J. A. Ajani, R. B. Catalano et al., "Recommended guidelines for the treatment of cancer treatmentinduced diarrhea," Journal of Clinical Oncology, vol. 22, no. 14, pp. 2918-2926, 2004.

[91] R. Sharma, P. Tobin, and S. J. Clarke, "Management of chemotherapy-induced nausea, vomiting, oral mucositis, and diarrhoea," The Lancet Oncology, vol. 6, no. 2, pp. 93-102, 2005.

[92] K. K. Hahn, J. J. Wolff, and J. M. Kolesar, "Pharmacogenetics and irinotecan therapy," American Journal of Health-System Pharmacy, vol. 63, no. 22, pp. 2211-2217, 2006.

[93] G. Richardson and R. Dobish, "Chemotherapy induced diarrhea," Journal of Oncology Pharmacy Practice, vol. 13, no. 4, pp. 181-198, 2007.

[94] D. Hind, P. Tappenden, I. Tumur, S. Eggington, P. Sutcliffe, and A. Ryan, "The use of irinotecan, oxaliplatin and raltitrexed for the treatment of advanced colorectal cancer: systematic review and economic evaluation," Health Technology Assessment, vol. 12, no. 15, pp. 1-182, 2008.

[95] P. Biason, S. Masier, and G. Toffoli, "UGT1A1*28 and other UGT1A polymorphisms as determinants of irinotecan toxicity," Journal of Chemotherapy, vol. 20, no. 2, pp. 158-165, 2008.

[96] G. E. Palomaki, L. A. Bradley, M. P. Douglas, K. Kolor, and W. D. Dotson, "Can UCT1A1 genotyping reduce morbidity and mortality in patients with metastatic colorectal cancer treated with irinotecan? An evidence-based review," Genetics in Medicine, vol. 11, no. 1, pp. 21-34, 2009.

[97] Z.-Y. Hu, Q. Yu, and Y.-S. Zhao, "Dose-dependent association between $\mathrm{UGT} 1 \mathrm{Al} * 28$ polymorphism and irinotecan-induced diarrhoea: a meta-analysis," European Journal of Cancer, vol. 46, no. 10, pp. 1856-1865, 2010.

[98] K.-I. Fujita and A. Sparreboom, "Pharmacogenetics of irinotecan disposition and toxicity: a review," Current Clinical Pharmacology, vol. 5, no. 3, pp. 209-217, 2010.

[99] M. M. Dias, R. A. McKinnon, and M. J. Sorich, "Impact of the UGT1A1 $* 28$ allele on response to irinotecan: a systematic review and meta-analysis," Pharmacogenomics, vol. 13, pp. 889899, 2012.

[100] Y. Kito and N. Teramoto, "Effects of Hange-shashin-to (TJ14) and Keishi-ka-shakuyaku-to (TJ-60) on contractile activity of circular smooth muscle of the rat distal colon," American Journal of Physiology, vol. 303, pp. 1059-1066, 2012.

[101] Y. Kase, T. Hayakawa, M. Aburada, Y. Komatsu, and T. Kamataki, "Preventive effects of Hange-shashin-to on irinotecan hydrochloride-caused diarrhea and its relevance to the colonic prostaglandin E2 and water absorption in the rat," Japanese Journal of Pharmacology, vol. 75, no. 4, pp. 407-413, 1997.

[102] Y. Kase, T. Hayakawa, A. Ishige, M. Aburada, and Y. Komatsu, "The effects of Hange-shashin-to on the content of prostaglandin E2 and water absorption in the large intestine of rats," Biological \& Pharmaceutical Bulletin, vol. 20, no. 9, pp. 954-957, 1997.

[103] Y. Kase, T. Hayakawa, S. Takeda, A. Ishige, M. Aburada, and M. Okada, "Pharmacological studies on antidiarrheal effects of Hange-shashin-to," Biological \& Pharmaceutical Bulletin, vol. 19, no. 10, pp. 1367-1370, 1996. 
[104] K. Takasuna, Y. Kasai, Y. Kitano et al., "Protective effects of kampo medicines and baicalin against intestinal toxicity of a new anticancer camptothecin derivative, irinotecan hydrochloride (CPT-11), in rats," Japanese Journal of Cancer Research, vol. 86, no. 10, pp. 978-984, 1995. 


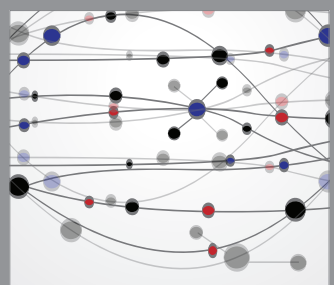

The Scientific World Journal
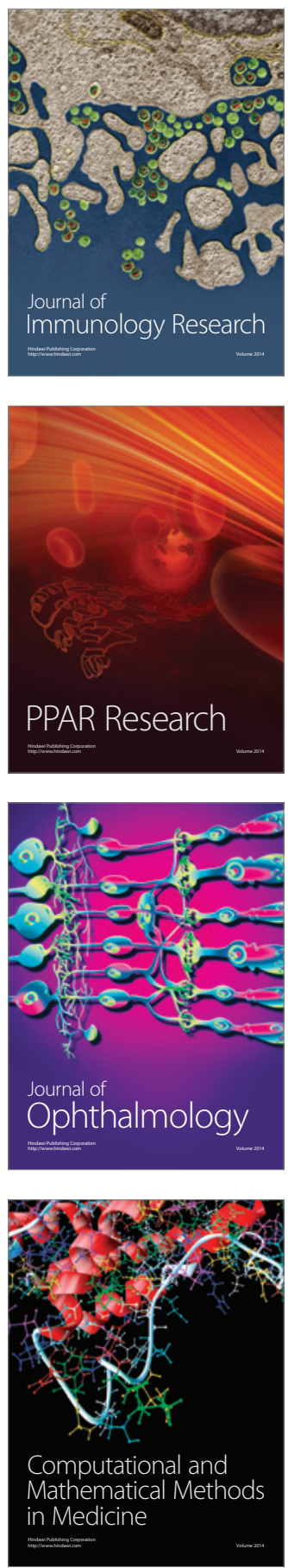

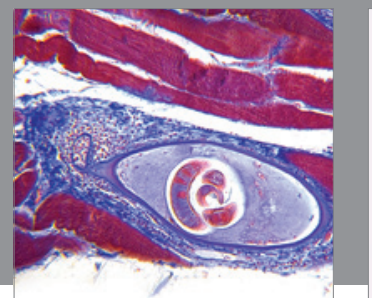

Gastroenterology

Research and Practice
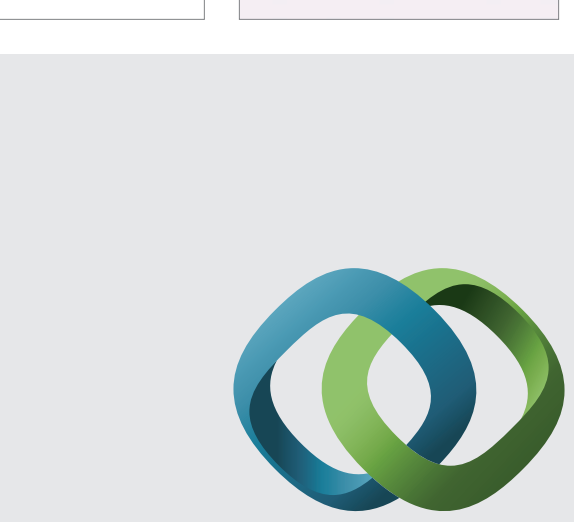

\section{Hindawi}

Submit your manuscripts at

http://www.hindawi.com
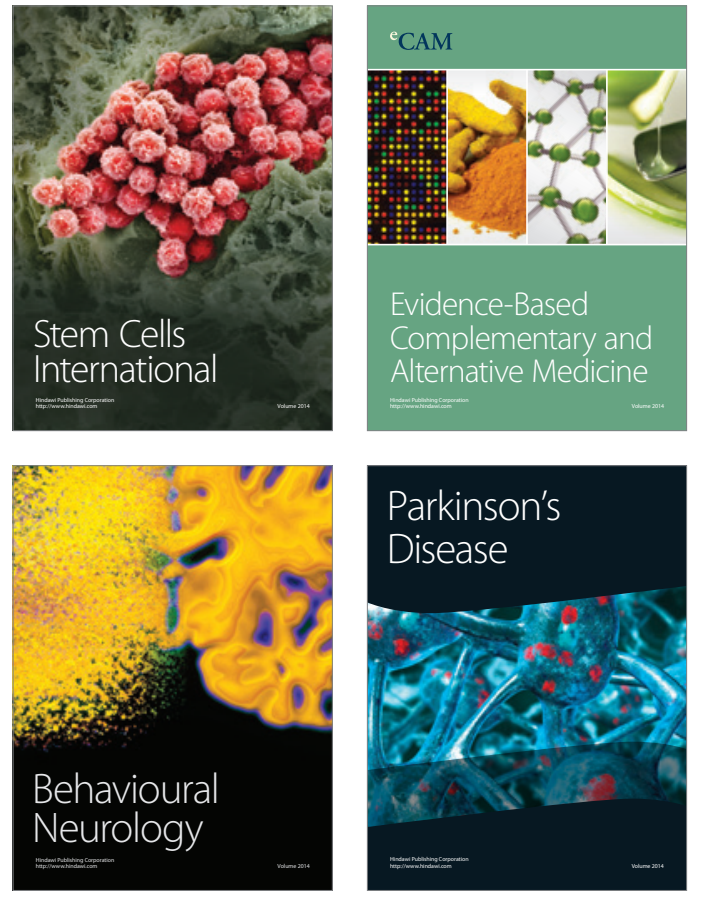
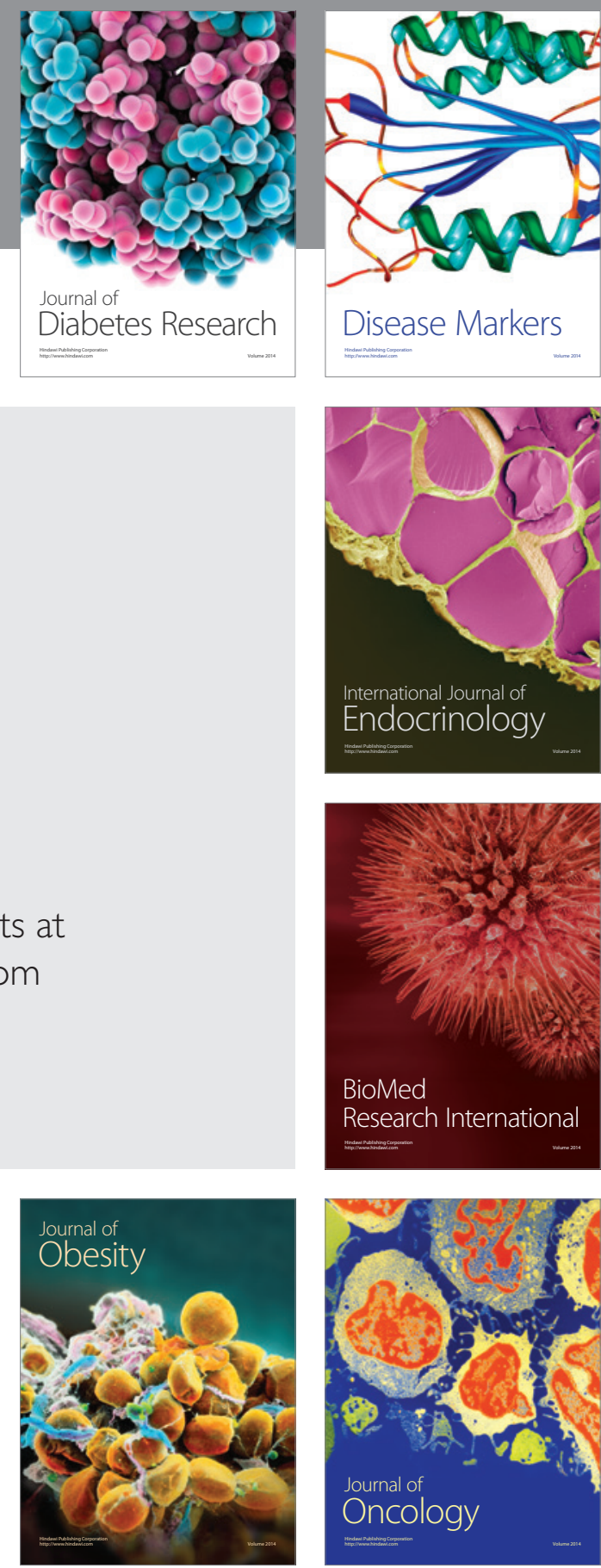

Disease Markers
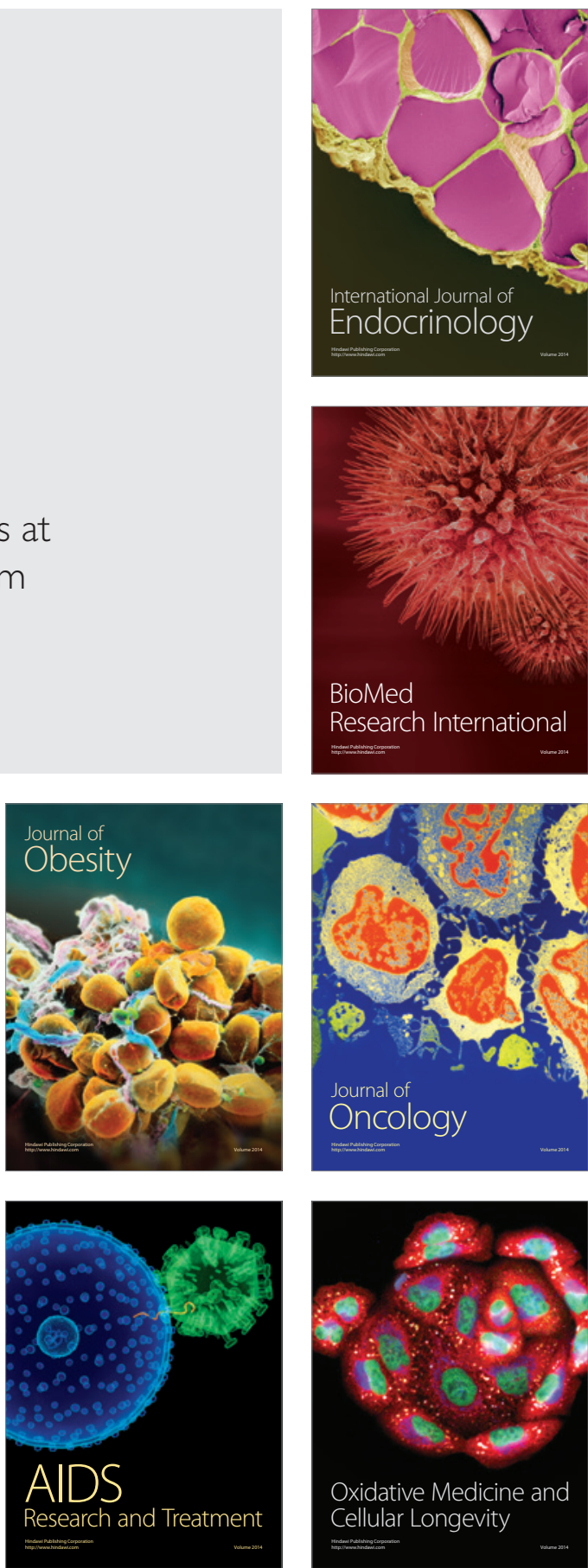